\title{
Helping Elderly Users Report Pain Levels: A Study of User Experience with Mobile and Wearable Interfaces
}

\author{
Iyubanit Rodríguez, ${ }^{1}$ Gabriela Cajamarca, ${ }^{1}$ Valeria Herskovic, \\ Carolina Fuentes, ${ }^{2}$ and Mauricio Campos ${ }^{3}$ \\ ${ }^{1}$ Department of Computer Science, Pontificia Universidad Católica de Chile, Santiago, Chile \\ ${ }^{2}$ School of Computer Science, University of Nottingham, Nottingham, UK \\ ${ }^{3}$ Medicine School, Pontificia Universidad Católica de Chile, Santiago, Chile \\ Correspondence should be addressed to Valeria Herskovic; vherskovic@uc.cl
}

Received 28 July 2017; Accepted 4 October 2017; Published 6 November 2017

Academic Editor: Pino Caballero-Gil

Copyright (C) 2017 Iyubanit Rodríguez et al. This is an open access article distributed under the Creative Commons Attribution License, which permits unrestricted use, distribution, and reproduction in any medium, provided the original work is properly cited.

Pain is usually measured through patient reports during doctor visits, but it requires regular evaluation under real-life conditions to be resolved effectively. Over half of older adults suffer from pain. Chronic conditions such as this one may be monitored through technology; however, elderly users require technology to be specifically designed for them, because many have cognitive and physical limitations and lack digital skills. The purpose of this article is to study whether mobile or wearable devices are appropriate to self-report pain levels and to find which body position is more appropriate for elderly people to wear a device to self-report pain. We implemented three prototypes and conducted two phases of evaluation. We found that users preferred the wearable device over the mobile application and that a wearable to self-report pain should be designed specifically for this purpose. Regarding the placement of the wearable, we found that there was no preferred position overall, although the neck position received the most positive feedback. We believe that the possibility of creating a wearable device that may be placed in different positions may be the best solution to satisfy users' individual preferences.

\section{Introduction}

Patient monitoring collects health information in real time [1], which can help health professionals improve treatment and diagnosis [2], while reducing health costs [3]. Chronic pain is described as "ongoing or recurrent pain, lasting beyond the usual course of acute illness or injury or more than 3 to 6 months, and which adversely affects the individual's well-being" [4]. Pain is frequent in the older population: up to $53 \%$ of elderly adults report suffering from recent pain [5]. Its treatment requires regular evaluation under reallife conditions in order to be resolved effectively [6]. Pain usually is measured through patients' self-reports only during medical appointments [7], using pain scales such as the Numerical Rating Scale or Verbal Rating Scale [8]. Pain is usually registered on paper, which can cause information loss and difficulties in analyzing and searching for data [9].
Users may be asked to report information remotely, for example, through the experience-sampling method, which asks participants to provide details about their current circumstances at certain intervals [10]. There have been several studies to explore whether elderly people self-report accurate information, with variable findings (e.g., $[11,12])$. For patients with pain, self-reporting is believed to help patients become more aware of the characteristics of their pain, for example, its intensity, patterns, triggers, and location [13] and be more engaged in the self-management activity [14].

A wearable device is a "computer that is always with you, is comfortable and easy to keep and use, and is as unobtrusive as clothing" [15]. A wearable device must meet the following criteria: (1) the device is attached to the body and the user does not need to hold it, (2) the user does not remove the device to perform tasks or actions, and (3) the user must not separate the device from the body to interact with it [16]. In addition, 
a wearable is on and working at all times [15]. Wearables can assist in monitoring patients with chronic pain during their daily routine, helping them better understand their illness and detect complications [17]. However, there is evidence of unequal access to technology, and, in some users, a lack of digital skills may hinder the possibility of using electronic devices for health monitoring [18]. The concept of wearability is used to describe how the wearable device interacts with the body [19] in terms of physical, emotional, and social comfort [20]. Designers of wearable devices must have knowledge of human physiology, since discomfort becomes evident when clothing impedes or restricts movement or visibility [21]. The placement of the device is one of the key concepts of wearability. The physiological, biomechanical, and comfort consequences should be included when evaluating a wearable device [16].

In this paper, we aim to explore the following research question: are wearable interfaces appropriate devices for elderly people to self-report their pain level?. To answer this question, we divided our research into two phases: the first aimed at understanding whether mobile devices or wearable devices are more appropriate for self-reporting pain levels and the second aimed at understanding which body position is more appropriate for elderly people to wear a device to self-report pain.

This paper is organized as follows. First, we discuss related work, especially focusing on existing technologies to report pain and studies on where wearable devices should be placed on the body. Then, Sections 3 and 4 describe each phase of the research, including the implemented prototypes, experiments, results, and discussion. Finally, Section 5 presents our conclusions and discusses limitations and possible avenues of future work.

\section{Related Work}

2.1. Technology and Interfaces for Elderly Adults. Aging is a process that depends on genetics, lifestyle, health [23], and gender, so the age in which a person is considered to be elderly varies. In several areas of the world, people over 50 are considered to be older adults [24], while the United Nations considers those over 60 to be older adults [25]. For the purposes of this study, and considering the cultural context of our study (conducted in Santiago, Chile), we consider people over 60 years of age and who are retired as elderly/older adults.

Elderly users require technology to be specifically designed for them, because many have cognitive and physical limitations [26], as well as a lack of digital skills that limits their ability to use electronic devices for disease monitoring [18]. Specifically in Chile, a high percentage of older adults have little experience with technology, almost $60 \%$ of 55 to 65-year olds in Chile have no computer experience whatsoever, while the average in OECD countries was $32 \%$ [27]. Regarding wearable devices, elderly users fear this type of technology may increase isolation and express concerns about safety and high costs [28]. Most studies with wearables and interaction have been conducted in countries in which older adults have a higher rate of digital skills (and a previous interest in technology is key in acceptance of wearables [29]), so our particular context provides additional challenges.
Several researchers have highlighted the importance of creating wearable technology for elders, for example, proposing six relevant considerations: motor, vision, eyeglasses, hearing, executive function, and memory [30]. Researchers have evaluated the use of commercial wearables by elderly adults (e.g., [31, 32]), finding them to be acceptable but require training for use. Less mainstream wearables (e.g., head-mounted displays) have been more complex for elders [33]. It is somewhat clear that established wearables such as activity trackers, that require little interaction from users, result in a better user experience than more experimental, niche wearables or than wearables that require users to interact with them.

2.2. Technology for the Self-Report of Pain. A system for patients to report pain from anywhere, at any time, can be used to monitor the evolution of pain [6]. Several applications allow people to report episodes of pain; for example, one mobile application displays a human figure and asks the user to indicate the position, intensity, and type of pain he/she feels $[34,35]$ or a web application that uses a combination of body diagrams and a Numerical Rating Scale, providing the transfer of patient information to health professionals [36].

Researchers have proposed several novel interfaces to self-report pain. A tangible device that allows users to easily record their pain using a six-level scale found that these types of pain-recording devices may decrease the pain experience [6]. Wearables with interactive displays allow users to input information [37]. For example, a wearable device to selfreport pain and emotional state found that it may help users improve their self-knowledge [38]. These types of applications allow new avenues of patient-doctor interaction [9], but adherence rates are often low [39] because some are burdensome [14], or not portable.

2.3. Placement of Wearable Devices on Body. The placement of wearable devices for elderly people is an important issue. A wearable device must allow easy access and handling [40], be discreet, ergonomic, and well affixed [17], and allow body movement [21] and visibility [41]. Placement should be in areas that are relatively the same size across adults (with a large surface area) and with low movement when the body is moving [19].

Regarding the best location of these devices in the body, eight possible areas for the unobtrusive placement of wearables have been identified, for example, collar area, rear of the upper arm, waist and hips, thigh, and top of the foot [19]. In the case of biomedical sensors and devices, they have been placed on headbands, helmets, belts, shoes, socks, bracelets, arm bands, and shirts [17]. Furthermore, large and curvilinear areas can be used in skin interfaces, for example, the back, the back of the hand, and the neck [42].

A recent study compared the placement of a wearable for elders on the wrist, upper arm, and neck, finding that the wrist was the best location (allowing the best viewing angle, a greater willingness to exhibit the device, and less anxiety) but that personal characteristics affected preferences [41]. Another study found that the wrist has the advantage that it is positioned approximately in the same place and orientation 


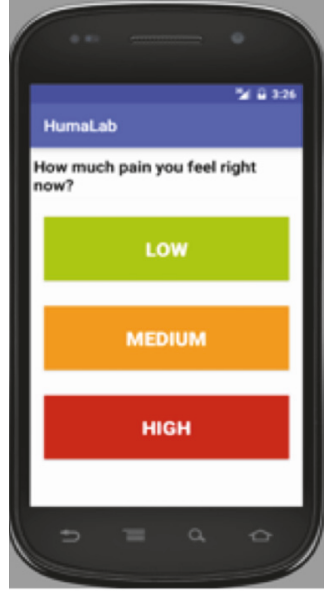

(a)

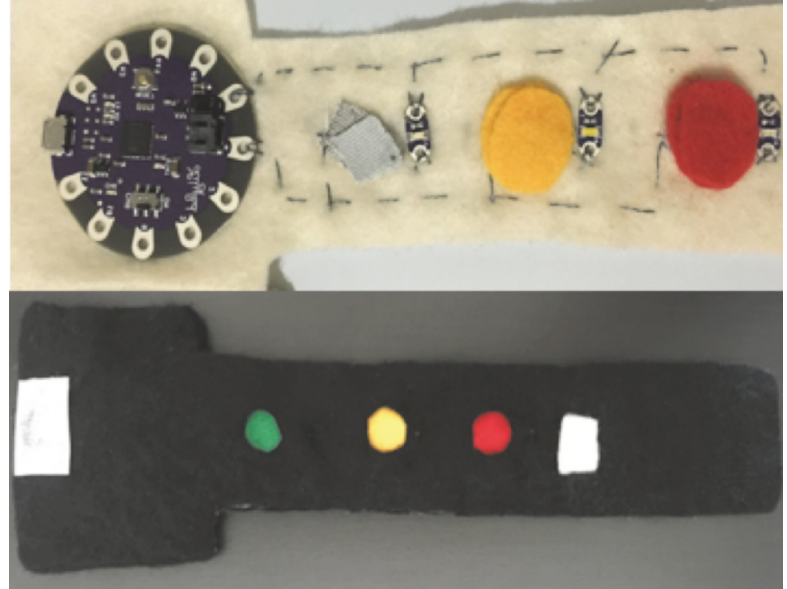

(b)

FIgure 1: (a) PainApp: mobile app. (b) B-pain: wearable device.

for all users [43]. Previous studies have not focused on where to place devices when elderly people have to interact with the device (through reporting information), which is the focus of this work.

\section{Phase I: Comparing Wearable Devices and Mobile Applications}

The first phase of this research focused on comparing two prototypes (one mobile and one wearable) that allow elders to self-report pain during their daily lives. A detailed account of the implemented devices and the experiment conducted in this phase can be found in [44]. Both of the devices use a simple Verbal Rating Scale with three levels of intensity (Low, Medium, and High). The two prototypes are described as follows:

(i) PainApp: it is a simple Android application that asks users about their pain level and stores the information in a database. The information can then be shared through email, Bluetooth, or social networks (Figure 1(a)).

(ii) B-pain: it is a bracelet-shaped wearable device, implemented using LilyPad Arduino. Pain is reported by pressing one of three buttons (green $=$ low; yellow $=$ medium; red $=$ high). The device provides feedback through a LED light (Figure 1(b)).

3.1. Materials, Methods, and Participants. The evaluation was done during May 2016. The participants were 12 undergraduate students ( 6 women and 6 men). The average age of participants was 26 (standard deviation $=5.4$ ). All participants had above basic digital skills. We applied semistructured interviews (each interview lasted about 15 minutes). To evaluate the solutions the participants interacted with the mobile application and wearable device and then we collected four types of information: (i) DIGCOMP: DIGCOMP is a standardized instrument to measure digital competences, where users are categorized into one of four possible groups, according to their digital skill levels: none, low, basic, or above basic [45].

(ii) System usability scale (SUS): SUS is a quick way to measure the overall usability of the system [46]. In this scale, scores below 60 indicate poor usability, while scores over 80 indicate very good usability [47].

(iii) Usability questionnaire: a questionnaire regarding usability of the wearable device.

(iv) Interview data: interviews were recorded (audio) and transcribed. Subsequently, each interviewee was assigned a code (P1 to P12).

3.2. Experiment. To avoid bias, half of the participants interacted with the mobile application first and the wearable device second, and the other half performed the opposite process. Each interview had the following structure:

(1) One researcher gave a brief introduction about the study and its purpose (5 minutes).

(2) The participant read a scenario describing a person with pain (a college student who after a car accident is suffering from chronic back pain) (5 minutes).

(3) The first interface was explained (3 minutes).

(4) The participant was given time to interact with the first interface (3 minutes).

(5) The researcher asked questions about the interface, using a predefined question set to guide the discussion (8 minutes).

(6) Steps (3)-(5) were repeated with the second interface (14 minutes).

(7) The researcher asked questions comparing the two interfaces (5 minutes). 
(8) Finally, participants completed the DIGCOMP, SUS, and user experience questionnaires ( 8 minutes).

3.3. Results. The interview data was transcribed, and thematic analysis was used for codification and analysis [48]. Some quotes from participants are provided in the results (translated from Spanish). We aimed to answer two questions: (1) which is more appropriate (has a higher rate of user acceptance) for monitoring pain: a wearable interface or a mobile application? And (2) which characteristics, or features, of the wearable interface, are critical for users to be able to report their pain levels?.

Regarding simplicity, $67 \%$ of participants found the wearable device (B-pain) was simpler, while only $16.5 \%$ found the mobile application simpler (and 16.5\% found that both technologies are equally simple). P4 said the following: "the bracelet, because it's easy and fast. If I feel pain I just need to push a button, while with the cellphone I have to turn it on, open the application, and then report pain." The wearable device was found by $75 \%$ of users to report pain at the right time, whereas in the mobile application the user was delayed by opening his/her phone and finding the app. Regarding the digital skills necessary to use each interface, $41.6 \%$ of participants believed the mobile application required some knowledge about how to use a smartphone, while $33 \%$ believed the wearable device only required a brief initial explanation about how to use it.

The key features of the wearable interface that are needed for users to be able to report their pain are the following ones:

(i) Low cognitive load: a device should require a low cognitive load and be simple to understand. For instance, B-pain has only one functionality. One user did mention that this device might not work for colorblind users, so it is important to complement the interface with, for example, words or textures that can help users with disabilities or other conditions use them.

(ii) Anytime/anywhere availability: a device to self-report pain should be easily available at all times, facilitating access to self-report. We call this anytimeanywhere availability "when you need it, you have it" (WYNIYHI).

(iii) Materials: it is important to consider the type of material with which the wearable device is designed. Materials can cause problems for users, for example, allergies.

(iv) Pain intensity and feedback: users should have the possibility of reporting several pain intensity levels (e.g., a 10-point numerical scale), but without making the design more complex. The feedback to the user should be clear and at the right time, so that the user is aware that the actions have been properly completed.

3.4. Discussion. Overall, this study found that both mobile and wearable applications may be appropriate for users to self-report pain, depending on the users and their context. For instance, in some situations, a wearable device may interfere with clothing conventions, and a mobile application (installed in a phone that the user would be carrying anyway) would be more appropriate. As evidence that pain-reporting applications are appropriate, a previous study found 25 diary or journal-type applications relating to pain, in which users could register their pain levels and optionally share data with health professionals and researchers [49]. However, in the current study, the wearable device was more widely accepted for monitoring pain than the mobile device.

As in previous studies, for example [50, 51], the fact that the wearable device had a clear purpose affected its acceptance. Although a wearable device to report pain would mean carrying an additional device, the surveyed users did not feel this was a limitation. The participants liked the immediate accessibility and limited functionality of the wearable device. One possible explanation is that users perceive mobile phones as a tool of social communication, while wearables are perceived as well-being devices [51].

The acceptance of a wearable device is affected by several social factors [52], among them perception of ease of use and mobility. Regarding ease of use, if a wearable device aimed at healthcare/well-being is perceived to be complicated, this could generate anxiety because a user might think that a mistake could be harmful to their health [52]. In the case of $B$ pain, the device is extremely simple and intuitive, serving only one function and with limited user interaction, which results in high ease of use. This ease of use has been found to give the user a feeling of control over the device [53]. Regarding mobility, although cellphones are highly personal and usually close to their user, B-pain is a bracelet, so it can also be carried anywhere easily. Since $B$-pain is worn on the wrist, similar to a watch or bracelet, it does not impede movement [19].

Previous research has found that portable devices should be lightweight, durable, and comfortable and with good appearance [51]; this was also evident in our study, as people were very emphatic in the importance of improving materials and aesthetics. Another aspect to consider in the appearance of wearable devices is the surrounding context and culture in which they are used, for example, the material, colors, and type of clothing may vary depending on the activity [21], so a wearable should be able to adapt to different contexts. The participants in our experiment mentioned that they would use both the wearable and the mobile application, since the phone may be more convenient for social situations, because it does not interfere aesthetically with the user's clothing.

This work found a trade-off between the usability of the wearable and the difficulty in replicating and maintaining it, since the mobile application is easily replicable and easily disseminated, while the portable device, although easier to use and requiring a lower cognitive load, requires hardware and must be built.

This phase of evaluation has several limitations that we would like to acknowledge. First, the participants were all students, so the digital skills and context are different from elders, and it is not possible to ascertain whether the results would have been the same if elders had been interviewed. The context of this study was a higher-education institution in Santiago, Chile, which could also account for cultural factors. Second, the participants did not use the device/application 


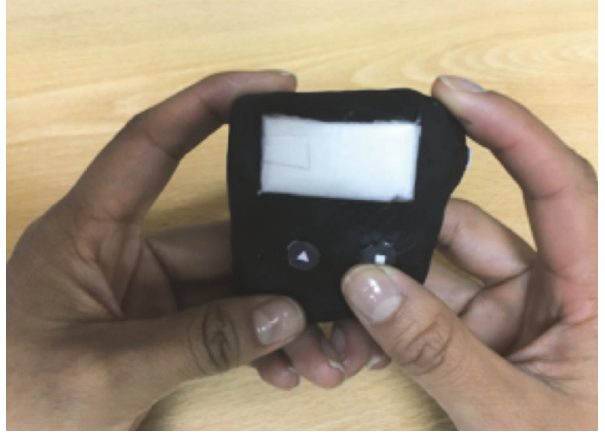

(a)

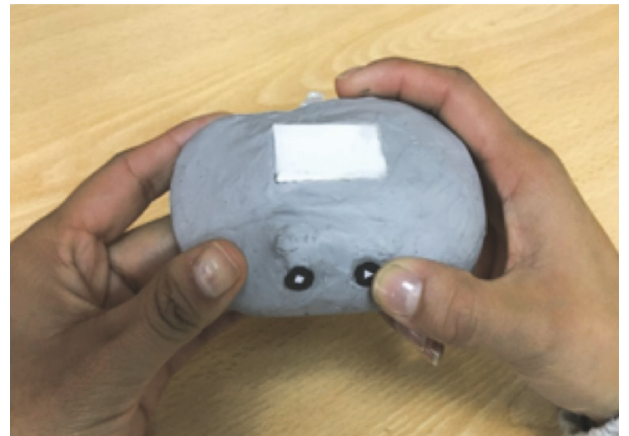

(b)

Figure 2: (a) Model A $(7 \times 5 \mathrm{~cm})$. (b) Model B $(6 \times 9 \mathrm{~cm})$.

during their daily life, rather they evaluated the interfaces after a brief period of interaction. Third, the participants were not users with pain, rather they were asked to imagine a context in which they were suffering from chronic pain and had to report it. Fourth, the size of the study was small, so we mainly focused on qualitative data. Although all of these limitations threaten the generalizability of our study, we found this first phase provided valuable insights, identifying concerns about how to design a wearable device that may allow elders to self-report pain, and we believe this is an important step before interviewing the actual targets of the device (elderly users).

\section{Phase II: RepWear (A Wearable to Self-Report Pain)}

Phase I found that a wearable device for self-report of pain is preferable over a mobile application, due to its simplicity, limited functionality, and greater accessibility. Therefore, since our goal was to design a device to self-report pain for elderly users, we set out to design a new prototype that could be used and tested by elderly users. In this phase, the focus was on the placement of a wearable device for elderly people to self-report their pain levels.

The design of this wearable device was based on several guiding principles: first, interfaces for elders should be simple and not use excessive instructions [54]. Second, the design should be based on a familiar concept to older adults [55]. For these reasons, and based on the previous study, we developed a simple, easy to use, intuitive prototype that requires little cognitive effort.

The wearable device we designed, called RepWear, allows self-reporting of pain by using two buttons: one allows navigating the intensity of pain from 0 to 10 , and the other one stores the selected value. In addition, a display shows the values of pain intensity and the device has an on/off switch. Two nonfunctional prototypes were created in order to understand people's perceptions about the preferred physical characteristics of such a device. Figure 2 shows two models that were made with modeling material and plastic 3D-printed pieces to simulate Arduino boards and buttons. We showed the prototypes to 10 adult participants ( 8 women,
2 men, average age: 37.2, SD: 19.98) and asked them to fill out a SUS questionnaire and participate in a brief interview. Nine out of 10 participants preferred Model A to Model B, stating that "this one is smaller, if it's big it scares me, simpler is better for an old man [like me]...." The results also showed a greater acceptability of Model A, since it received a SUS score of 90, while Model B had a score of 77 . The participants gave reasons for their selection such as the location of the buttons, the position of the switch, and the size, while recommending that (1) the buttons must have different colors, while keeping the icons and (2) the shape should be more curved. Finally, the participants mentioned that the positions where the device could be placed on the body were wrist, neck, waist or belt, arm, and pants pocket or/and shirt pocket.

Therefore, based on the previous results, a new, functional prototype was designed, incorporating a Numerical Rating Scale (0 to 10), anytime/anywhere availability, softer material, feedback through sound and touch, and a curved shape with colored buttons. The device was implemented using a LilyPad Arduino Main Board (electronic card based on ATmega168V) and a Lithium Ion Battery, $1 \mathrm{Ah}$. A Real-Time Clock (RTC) (DS1307) was used to record the time and date when the user self-reports pain intensity. The device incorporated two buttons: a red one to allow users to save pain intensity and a green one to allow users to select a pain intensity. The user may see the reported number on the display (Grove, 4 -Digit Display module). When users self-report pain, they receive feedback because the button is mechanical and clicks. Figure 3 shows a user self-reporting pain. The information (time and date, pain intensity) is saved in a microSD card. A miniature DPDT slide switch may be used to turn the device on or off.

4.1. Materials, Methods, and Participants. The evaluation was done during June and July 2017. The participants were 18 elderly people ( 13 women and 5 men). The participants were all older adults, ranging in age from 60 to 93 (average: 69.3, SD: 9.36). They did not have any mental disabilities. Table 1 describes each study participant. Participants with None digital skills mentioned that they only use the phone to call and/or send messages. In addition, two of the participants (P4 and P5) walk with support: one with a walker and the 
TABLE 1: Description of study participants.

\begin{tabular}{|c|c|c|c|c|c|c|c|c|}
\hline $\mathrm{P}$ & Age & Gender & Occupation & Educational level & Digital skills & Location of residence & Bedridden & Pain location \\
\hline $\mathrm{P} 1$ & 60 & $\mathrm{~F}$ & Housewife & High school & None & Own house & & Knee \\
\hline P2 & 82 & $\mathrm{~F}$ & Housewife & High school & None & Nursing home & $\bullet$ & Back \\
\hline P3 & 93 & M & Mechanic & Primary & None & Nursing home & $\bullet$ & Prostate \\
\hline P4 & 73 & M & Teacher & Master & Above basic & Nursing home & & Hands and legs \\
\hline P5 & 80 & $\mathrm{~F}$ & Housewife & Primary & None & Nursing home & & Legs \\
\hline P6 & 70 & $\mathrm{~F}$ & Teacher & University & Basic & Own house & & Back \\
\hline P7 & 65 & $\mathrm{~F}$ & Paramedic & Technical & Basic & Own house & & Back \\
\hline P8 & 60 & $\mathrm{~F}$ & Therapist & Technical & Above basic & Own house & & Back \\
\hline P9 & 62 & M & Grocer & High school & Basic & Own house & & Back \\
\hline P10 & 76 & $\mathrm{~F}$ & Housewife & High school & None & Own house & & Hip \\
\hline P11 & 79 & M & Building & None & None & Own house & & Neck \\
\hline $\mathrm{P} 12$ & 60 & M & Teacher & University & Basic & Own house & & Back \\
\hline P13 & 60 & $\mathrm{~F}$ & Secretary & Technical & Basic & Own house & & Back and foot \\
\hline P14 & 62 & $\mathrm{~F}$ & Housewife & High school & None & Own house & & Back and foot \\
\hline P15 & 65 & $\mathrm{~F}$ & Housewife & School & None & Own house & & Breast \\
\hline P16 & 72 & $\mathrm{~F}$ & Secretary & Technical & None & Own house & & Back and stomach \\
\hline P17 & 68 & $\mathrm{~F}$ & Housewife & School & None & Own house & & Column and knee \\
\hline P18 & 60 & $\mathrm{~F}$ & Housewife & High School & None & Own house & & Neck and hand \\
\hline
\end{tabular}

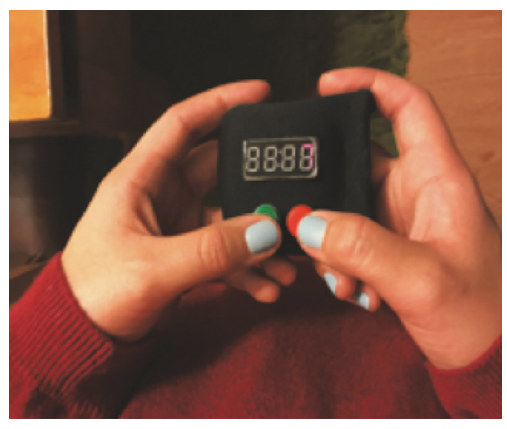

(a)

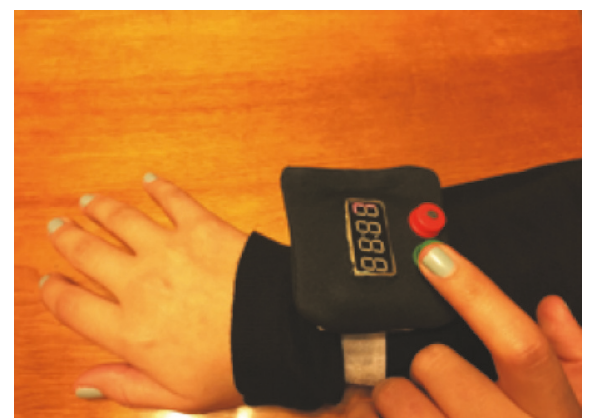

(b)

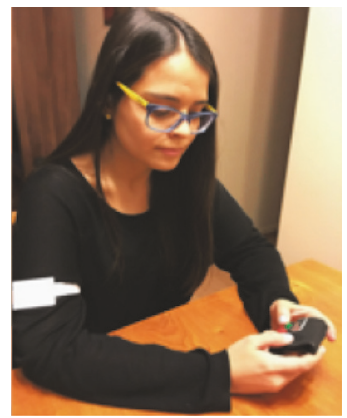

(c)

Figure 3: (a) RepWear prototype $(6 \times 6.3 \mathrm{~cm})$. (b) Self-report pain from the device on the wrist. (c) Extracted from the strap to self-report.

other with the help of another person. Two participants (P2 and P3) were bedridden.

To evaluate the placement of the device, the participants interacted with the device and placed it in 4 different body positions: wrist, waist, neck, and arm. The following four types of information were collected:

(i) DIGCOMP: DIGCOMP is digital skills questionnaire (see Section 3.1).

(ii) Wearable satisfaction questionnaire: we created a 7point Likert scale questionnaire based on the findings of a previous study on placement of wearables for elderly people [41]. We created one question for each of the following issues: willingness to show the wearable device, anxiety, oddness, fear of others' negative reaction, comfort, readability of the device screen, and unobtrusiveness for daily activities. (iii) AttrakDiff questionnaire: AttrakDiff is a questionnaire used to understand how users personally rate the usability and design of an interactive product. AttrakDiff has four dimensions: pragmatic quality, which is the ease with which the user can complete the task, hedonic quality-identity (HQ-I), which is the message that is communicated to others while the product is being used, hedonic quality-stimulation (HQ-S), which represents whether the development of user skills is encouraged, and attraction, or the overall charm of the product. Answers are on a scale of -3 to 3 (0 represents neutrality) [22, 56].

(iv) Interview data: each interview was recorded (audio), transcribed, and assigned a code (P1 to P18).

4.2. Experiment. To understand the perceptions of older adults about the placement of a wearable device to self-report pain, we chose 4 body parts to investigate: the wrist, arm, 


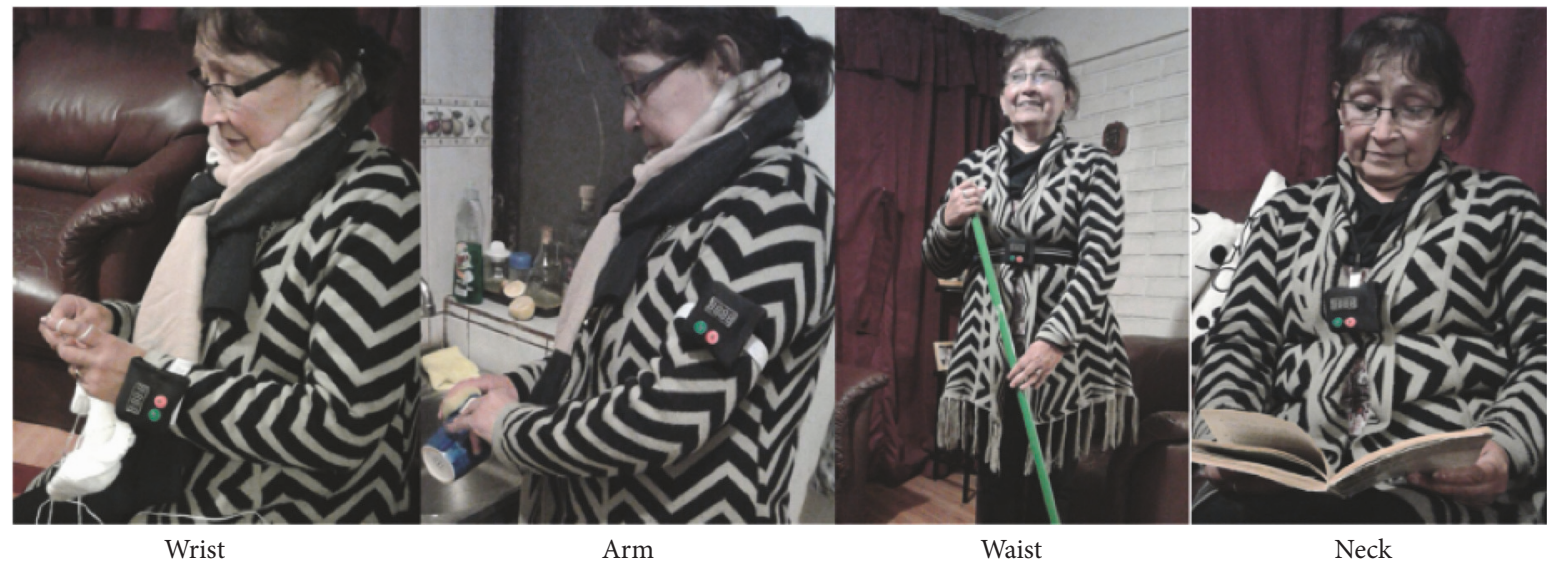

FIGURE 4: Use of RepWear while doing activities of daily living in the four body positions: wrist, arm, waist, and neck, respectively.

waist, and neck. The experiment had a duration of between 60 and 75 minutes per participant. The activities carried out were the following ones:

(i) The researcher explained the purpose of the investigation and answered questions about it, and then the participant signed the informed consent form (10 to 15 minutes).

(ii) The participant was asked basic information such as age, profession, education level, and pain location. They completed the DIGCOMP questionnaire regarding digital skills (5 minutes).

(iii) The participant used the device to self-report pain at each position of the body for 6 minutes (24 minutes for all four positions), while they performed their everyday routines and tasks (this process is shown in Figure 4). The researcher observed and took notes. Participants were asked to self-report pain once for each position, either directly from the device or by removing it from its strap and then returning it to the strap (see Figures 3(b) and 3(c)).

(iv) After completing each position, the participant answered the wearable satisfaction questionnaire.

(v) After completing all the positions, the researcher conducted a semistructured interview to collect information from participants about the preferences of the body positions to carry the device (ranking) and their perception about the device (5 to 10 minutes).

(vi) Finally, the participant completed the AttrakDiff questionnaire about RepWear (10 to 15 minutes).

\subsection{Results}

4.3.1. Body Placement Results. Participants were asked to rank their preferred placements for the device. One of the participants chose not to provide a ranking, as he/she felt all the positions were approximately the same. The ranking data was analyzed using the Shapiro-Wilk test, obtaining that the distribution is not normal $(\alpha=0.05)$. Then, the KruskalWallis test was applied $(n=17)$, with the result that the ranking of positions (neck, arm, waist, and wrist) did not have a statistically significant difference overall. However, we analyzed the qualitative responses in depth to understand the user experience with each possible body position. Next, we provide the analysis of each position. Quotes from the interviews are provided, translated from Spanish.

(i) Neck: the neck position was comfortable and loose for the participants "because... let's see, it did not bother me at all, really at all. It's like wearing a necklace: more comfortable, friendlier". Also, the readability of the screen was good: "it's comfortable, it's not bothersome, because from here I can see [the pain scale]."

(ii) Wrist: responses about this position were polarized. Some felt that wearing the device on the wrist interfered with their activities: "it is closer to the hand with which I do things... it could bother me when grabbing things or separating [papers]. It would disturb me more", or were worried about it falling or becoming damaged "it may inconvenience me more, because of where it is placed, I could hit it on something", "it is uncomfortable because we are moving our hands at all times and it may fall. I can do things but it may fall. That's the danger I see." Other participants gave positive comments to the position of the wrist as being adequate to see the pain scale: "it's more comfortable to manipulate the device and look at it [the pain scale]" and for comfort "...more comfortable to move... for mobility."

(iii) Arm: this position had very few comments from the participants. People who ranked it first thought that it was comfortable and those who ranked it last thought the opposite: "because of my usual activities, it would be uncomfortable: to throw a ball, to jump...". Users also thought they would have mobility problems "it's more uncomfortable. . it would not allow me to move".

(iv) Waist: the waist was the position that had the most negative comments. Participants emphasized that readability is low, since in this position the device could be covered by clothes: "I always wear something 


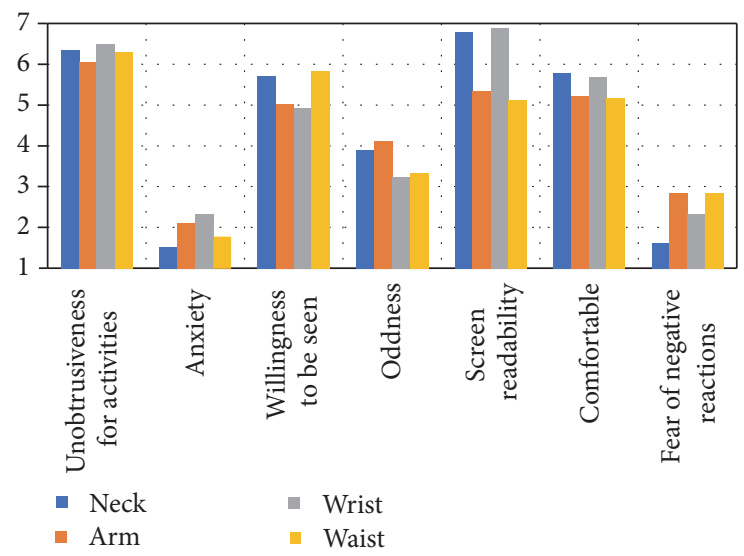

FIGURE 5: The average satisfaction degree in four parts of the body (units = points, 1 (strongly disagree) to 7 (strongly agree)).

that covers my waist. Even though I am thin I feel more secure with my waist hidden from view and compressed." Another participant indicated that the waist position did not adjust to heavier shapes: "I am bigger and fat." However, three people mentioned that the waist is comfortable to report the pain and that the device will be in less danger: "I can put it in the front and it will be more protected, I'll take better care of it."

The results from the wearable satisfaction questionnaire (Figure 5) for each position of the body regarding unobtrusiveness, anxiety, willingness to be seen, oddness, screen readability, comfort, and fear of negative reactions show that participants mostly gave similar scores to each position and that the results were positive. For example, users felt that the device was unobtrusive (average score: 6.29) and caused low anxiety (average score: 1.93), they were willing to be seen (average score: 5.83), and it was more or less odd (average score: 3.64 ), readable (average score: 6.03), and comfortable (average score: 5.46) and did not cause much fear of negative reactions (average score: 2.40 ).

These responses were analyzed using the Shapiro-Wilk test $(\alpha=0.05)$, finding that the responses did not follow a normal distribution. Then, the Kruskal-Wallis test was performed ( $n=18, \alpha=0.05$ ), finding that the only statistically significant difference was the readability of the device $(p$ value: 0.0358). Then, we applied the Mann-Whitney test, finding that the neck and wrist had significantly higher scores than the waist for readability ( $p$ values: 0.0227 and 0.004 ).

We discuss each of the items as follows:

(i) Willingness to show the wearable device: participants said that they would show the device in public only if it was absolutely necessary: "I would use it only if I felt a lot of pain." One participant mentioned that wearing the device on the waist may help the device be unnoticed.

(ii) Fear of others' negative reaction: all the participants felt little fear of the reaction of others when carrying the device: "at this age I don't care what others think of me, I am too old for that [laughing]." Also, three

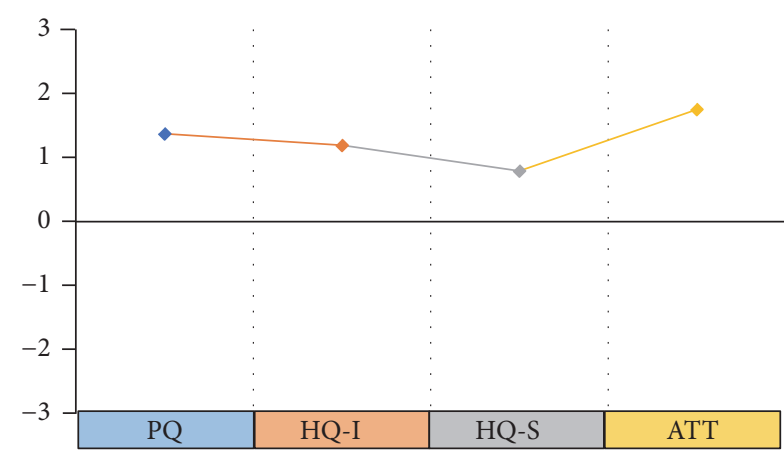

FIGURE 6: Average values for pragmatic quality (PQ), hedonic quality-identification (HQ-I), hedonic quality-stimulation (HQ-S), and attractiveness (ATT) (results from [22]).

participants commented that they would fear that the device could be stolen.

(iii) Readability of the device screen: the positions that offer a better view of the pain scale are the wrist and neck with a score of 6.89 and 6.78 , respectively. This is because in those positions people can self-report pain without removing the device from the strap, whereas in the arm (5.33) and the waist (5.11) the majority of the participants (13 people) chose to remove the device from strap to (1) have a better view of the scale and (2) be able to handle the button.

(iv) Anxiety: the reported scores for anxiety were very low; that is, the participants did not feel anxious when using the device.

(v) Oddness: participants did not feel odd when using the device, rather they felt that others might: "people could feel strange when they see a device like this...." The participants felt that wearing the device on the wrist was not odd because of the similarity of the device with a wristwatch: "for me, it's like wearing a watch, for that reason I liked it more, because I am used to it."

(vi) Comfortable: the majority of the participants were comfortable using the device in the 4 parts of the body; however, the neck (5.78) and the wrist (5.67) received the highest scores, "I feel I can wear it comfortably and securely."

(vii) Unobtrusiveness for daily activities: participants used the device to perform their daily activities to determine which position was most suitable for that purpose. The results showed that when the device was placed on the wrist (6.50), there were fewer interruptions or discomfort.

4.3.2. User Experience Results. User experience (UX) is defined as a "person's perceptions and responses resulting from the use and/or anticipated use of a product, system or service" [57], including aspects of product use, reflections of interactions, user expectations, and feelings [58]. User experience was measured through the AttrakDiff questionnaire (Figure 6). RepWear was found to have a high pragmatic 


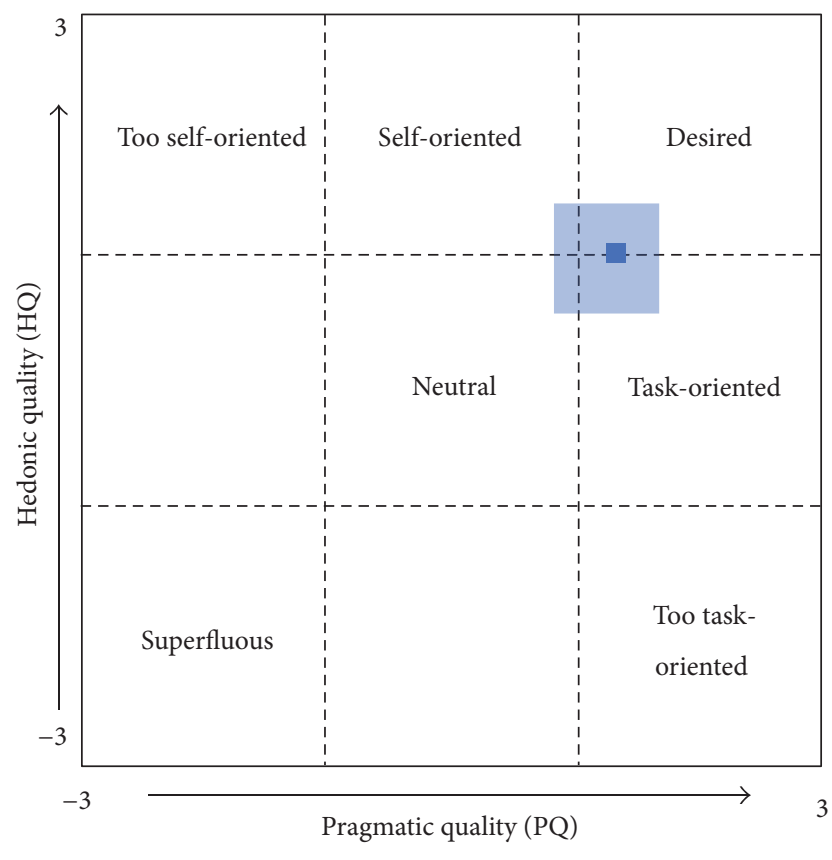

Figure 7: Portfolio with average values of the dimensions PQ and HQ and the respective confidence rectangle (results from [22]).

quality (PQ $=1.37$ ), which means users can do the task of self-reporting pain. Nevertheless, hedonic quality was low $(\mathrm{HQ}=0.99)$ leaving the device in between the task-oriented and desired categories (Figure 7). The confidence levels were 0.46 for PQ and 0.48 for HQ, which means that answers were a little scattered. The attractiveness dimension (ATT) had a score of 1.75 , which means that participants had a positive assessment of the appeal of the device. The score for hedonic quality-identification (HQ-I), or "how others can see the product," was 1.19. The hedonic quality-stimulation (HQS) score was the lowest (0.79), meaning that RepWear was not perceived as a device that helps to develop personal skills and improve knowledge. Figure 8 shows that the word pairs undemanding-challenging and cautious-bold, pertaining to the HQ-S, had scores below 0 .

During the interviews, participants were asked their opinions about the device. They mentioned that RepWear was easy to use, handy, and useful: "I found it was novel, and good in the sense that one can have it on all day to mark pain intensity and the time it happened." Also, they thought the pain scale was appropriate: "it's good that it asks me on a scale from 0 to $10 \ldots$ it's much better." Another participant mentioned that the colors on the buttons reminded her how to use the device: "... I liked the colors from these two little things, it's like calling on the phone."

Regarding the aspects of RepWear that participants did not like, four mentioned that they would prefer a thinner, lighter device: "it protrudes too much, it should be smaller, more adaptable to the body." Nine participants said there was nothing that they did not like about the device. Additionally, one participant expressed that he did not like the device at all because it was not useful.
One participant thought that the buttons were too sensitive and suggested improvements: "the numbers, when I push it's too sensitive, it changes way too fast." Another participant would like the device to be more firmly attached to the body: "it should be fixed to a spot, because it's not secure... maybe a strap would make it more secure. This could fall." Participants also commented on the device material and color, with some suggesting different colors or using plastic material.

4.4. Discussion. Our results suggest that the neck may be the best position for a wearable to self-report pain, since it generally had good scores in all of the wearable questionnaire items (especially regarding anxiety, fear of negative reactions, screen readability, and comfort). These results differ from previous results that suggested that a neck wearable device would be annoying and uncomfortable [41]. This may be because of cultural reasons or because RepWear required selfreport, so this potential interaction may affect how users perceive the device. Further study is needed to examine these discrepancies and understand the reasons behind them.

Our device, although designed as a wearable, did not completely fulfill one of the criteria for wearables, that is, that the device does not need to be separated from the body for interaction. When the device was worn in the waist and arms, the participants chose to remove it to manipulate it, changing the device from a wearable to a portable device. This allows us to identify that placement is key: the position of the device at the body has an impact into facilitating or hindering visualization and self-report.

The AttrakDiff questionnaire measures hedonic qualities such as stimulation and innovation. In the case of an interface designed for elderly users with low digital skills, we wanted to create an interface that was not intimidating. In this sense, the low HQ-S results may be interpreted in a positive light, since they are related to adjectives such as undemanding and cautious, showing that the users did not feel threatened or challenged by the device. We believe adding buttons that clicked mechanically was partially the cause of this result. Previous studies have found that elders feel computer anxiety [59] about damaging a technological device while using it, anxiety about "doing something wrong." For example, elderly users are anxious when moving a computer mouse [60]. However, in our study, even elders with no digital skills did not feel anxious, except when using the device on the wrist, which they felt could damage the device (e.g., when washing dishes).

It is important to mention that the evaluated device was a prototype, lacking the more polished look and feel of a finished product, as well as being bulkier. These characteristics of the prototype could influence the perception of the participants when trying on the device. Generally, when designing devices such as this one, the thickness of the device should be minimized, allowing greater safety and comfort [19]. Our prototype had a thickness of $2.5 \mathrm{~cm}$, which may cause some discomfort in the participant if it is worn on certain parts of the body such as the wrist. Also, elders tend to prefer a compact device for portability [41] and movement [52]. In this regard, the RepWear prototype still has room 


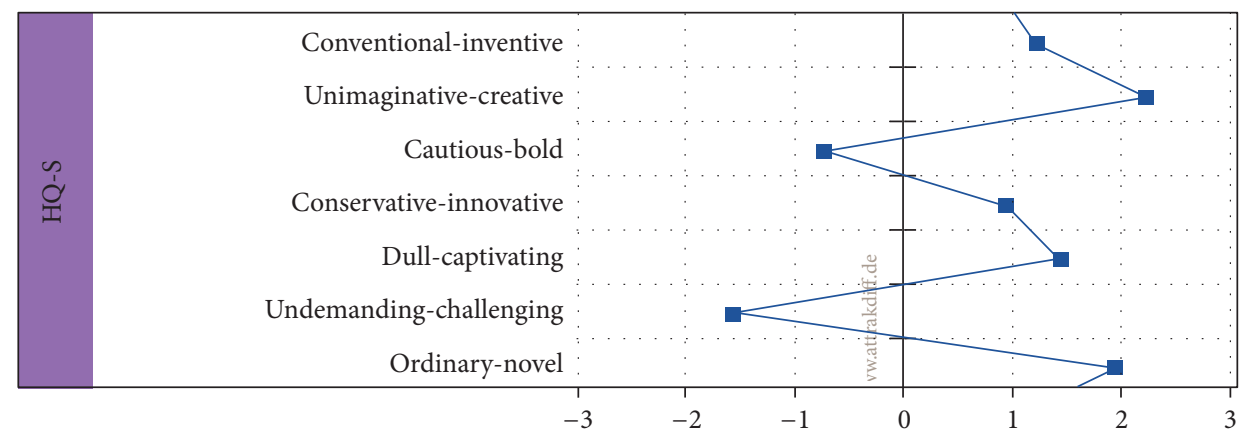

FIGURE 8: The mean values of the word pairs of hedonic quality-stimulation (HQ-S) (diagram from [22]).

for improvement and size, thickness, and weight should be further decreased.

In this phase the participants were elderly adults, which allows for some comparison with the first phase of our research. We found that the elderly users were less concerned with aesthetics; they did not find the device overly odd nor feared negative reactions, and they did not comment extensively on materials nor matching clothing as the participants in the first phase. Rather, the elderly participants mainly focused on the functionality and comfort of the device, especially in regard to being able to carry out their activities normally. The literature has also found perceived utility to be of great importance for elders, since they will use a system if they consider it to be useful and reliable and provide benefits to their independence [52]. In our study, only one participant (out of 18) did not find the device to be useful. Finally, the cost of a device can influence that user to use it, and an important challenge is for technology to be of low-cost [61]. Our device is of low-cost, using simple components (and would be extremely low-cost if it was mass-produced).

We would like to acknowledge the study limitations for this phase. First, the number of participants in the study was small, because of the difficulty in recruiting elderly participants, since we only chose to interview those without cognitive impairments, and the extensive questionnaires we used caused the participants some cognitive overloading, causing them to become tired. This may suggest the need for using questionnaires that are especially designed for the elderly, in order to improve their willingness to participate. Second, the study was conducted in Santiago, Chile, and cultural characteristics may make its results not generalizable to a broader region.

\section{Conclusion and Future Work}

The goal of this research was to explore the best way for older adults to report pain. To achieve this goal, we divided our research into two phases: (1) finding out whether a mobile or wearable interface would be more appropriate and (2) finding where to place the wearable device.

First, we found that users preferred the wearable device over the mobile application and that a wearable to self-report pain should be designed specifically for this purpose, be aesthetically pleasing, and allow users to report easily and at the right time. Second, we found that although participants had individual preferences for the placement of the wearable device, there was no preferred position overall. The neck position received the most positive feedback, because it produced less anxiety, there was no fear of the reaction of other people, and it was comfortable and had a good view of the screen. We believe that the possibility of creating a wearable device that may be placed in different positions may be the best solution to satisfy users' individual preferences.

We would like to acknowledge some of the study limitations. First, the study should be expanded to include a higher number of participants, although there are several challenges in recruiting older participants without cognitive impairment, and extensive questionnaires were found to cause some cognitive overloading. Second, the first phase of the study used student participants (due to the difficulties in recruiting elderly people), and these results may therefore not completely be applicable to the elderly. Third, the study was conducted in Santiago, Chile, and cultural characteristics may not make the results generalizable to a broader region.

As future work, we will analyze how to improve certain physical aspects of the device (e.g., decreasing size and thickness), in order to make the device more comfortable and versatile. We will also work on recruiting additional participants to explore in more depth the reasons behind their placement preferences.

\section{Conflicts of Interest}

The authors declare that there are no conflicts of interest regarding the publication of this paper.

\section{Acknowledgments}

This project was supported partially by CONICYT-PCHA/ Doctorado Nacional, 2014-63140077, CONICIT and MICIT Costa Rica Ph.D. scholarship grant, Universidad de Costa Rica, and CONICYT/FONDECYT no. 1150365 (Chile). The authors thank Javiera Rivas, Leonor Toro, and Teresa Uribe for their help with the experiment and the nursing homes that opened their doors to them. 


\section{References}

[1] World Health Organization, mHealth: New Horizons for Health through Mobile Technologies, vol. 3 of Global Observatory for eHealth Series, Geneva, Switzerland, 2011.

[2] A. Alahmadi and B. Soh, "A smart approach towards a mobile e-health monitoring system architecture," in Proceedings of the 2011 International Conference on Research and Innovation in Information Systems, ICRIIS'11, November 2011.

[3] M. J. Field and J. Grigsby, "Telemedicine and Remote Patient Monitoring," Journal of the American Medical Association, vol. 288, no. 4, pp. 423-425, 2002.

[4] ACPA., Glossary @ONLINE, 2016.

[5] K. V. Patel, J. M. Guralnik, E. J. Dansie, and D. C. Turk, "Prevalence and impact of pain among older adults in the United States: findings from the 2011 National Health and Aging Trends study," PAIN, vol. 154, no. 12, pp. 2649-2657, 2013.

[6] I. Alakärppä, J. Riekki, and R. Koukkula, "Pervasive pain monitoring system user experiences and adoption requirements in the hospital and home environments," in Proceedings of the 2009 3rd International Conference on Pervasive Computing Technologies for Healthcare - Pervasive Health 2009, PCTHealth 2009, pp. 1-8, April 2009.

[7] A. Chhikara, A. Rice, A. McGregor, and F. Bello, "In-House Monitoring of Low Back Pain Related Disability (IMPAIRED)," in Proceedings of the 2008 30th Annual International Conference of the IEEE Engineering in Medicine and Biology Society, pp. 4507-4510, August 2008.

[8] A. Williamson and B. Hoggart, "Pain: a review of three commonly used pain rating scales," Journal of Clinical Nursing, vol. 14, no. 7, pp. 798-804, 2005.

[9] F. Spyridonis, J. Hansen, T.-M. Grønli, and G. Ghinea, "PainDroid: An android-based virtual reality application for pain assessment," Multimedia Tools and Applications, vol. 72, no. 1, pp. 191-206, 2014.

[10] R. Larson and M. Csikszentmihalyi, "The experience sampling method," New Directions for Methodology of Social \& Behavioral Science, 1983.

[11] T. L. Bush, S. R. Miller, A. L. Golden, and W. E. Hale, "Selfreport and medical record report agreement of selected medical conditions in the elderly," American Journal of Public Health, vol. 79, no. 11, pp. 1554-1556, 1989.

[12] K. Hilton, J. Fricke, and C. Unsworth, "A comparison of selfreport versus observation of performance using the assessment of living skills and resources (ALSAR) with an older population," The British Journal of Occupational Therapy, vol. 64, no. 3, pp. 135-143, 2001.

[13] C. Rini, D. A. Williams, J. E. Broderick, and F. J. Keefe, "Meeting them where they are: Using the Internet to deliver behavioral medicine interventions for pain," Translational Behavioral Medicine, vol. 2, no. 1, pp. 82-92, 2012.

[14] H. MacLeod, A. Tang, and S. Carpendale, "Personal informatics in chronic illness management," in Proceedings of the Graphics Interface GI '13, pp. 149-156, Canadian Information Processing Society, Toronto, Canada, 2013.

[15] B. J. Rhodes, "Wearable remembrance agent: a system for augmented memory," in Proceedings of the 1997 1st International Symposium on Wearable Computers, pp. 123-128, October 1997.

[16] J. F. Knight, D. Deen-Williams, T. N. Arvanitis et al., "Assessing the wearability of wearable computers," in Proceedings of the 10th IEEE International Symposium on Wearable Computers, ISWC 2006, pp. 75-82, October 2006.
[17] A. Dittmar, R. Meffre, F. De Oliveira, C. Gehin, and G. Delhomme, "Wearable medical devices using textile and flexible technologies for ambulatory monitoring," in Proceedings of the 2005 27th Annual International Conference of the Engineering in Medicine and Biology Society, IEEE-EMBS 2005, pp. 7161-7164, September 2005.

[18] S. Hamine, E. Gerth-Guyette, D. Faulx, B. B. Green, and A. S. Ginsburg, "Impact of mHealth chronic disease management on treatment adherence and patient outcomes: a systematic review," Journal of Medical Internet Research, vol. 17, no. 2, p. e52, 2015.

[19] F. Gemperle, C. Kasabach, J. Stivoric, M. Bauer, and R. Martin, "Design for wearability," in Proceedings of the Second International Symposium on Wearable Computers, Digest of Papers, pp. 116-122, October 1998.

[20] L. E. Dunne and B. Smyth, "Psychophysical elements of wearability," in Proceedings of the 25th SIGCHI Conference on Human Factors in Computing Systems 2007, CHI 2007, pp. 299-302, New York, NY, USA, May 2007.

[21] J. McCann, R. Hurford, and A. Martin, "A design process for the development of innovative smart clothing that addresses enduser needs from technical, functional, aesthetic and cultural view points," in Proceedings of the 9th IEEE International Symposium on Wearable Computers, ISWC 2005, pp. 70-77, October 2005.

[22] Attrakdiff, http://attrakdiff.de/index-en.html.

[23] S. Singh and B. Bajorek, "Defining 'elderly' in clinical practice guidelines for pharmacotherapy," Pharmacy Practice, vol. 12, no. 4, article no. 489, 2014.

[24] World Health Organization, "Proposed working definition of an older person in Africa for the MDS project," http://www.who .int/healthinfo/survey/ageingdefnolder/en/.

[25] F. J. García-Peñalvo, M. Á. Conde, and V. Matellán-Olivera, "Learning and collaboration technologies. technology-rich environments for learning and collaboration: hci international," in Mobile Apps for Older Users - The Development of a Mobile Apps Repository for Older People, vol. 8524, pp. 117-126, Springer International Publishing, Cham, Switzerland, 2014.

[26] L. Qingchuan and L. Yan, "Older adults and digital technology: A study of user perception and usage behavior," in Proceedings of the AHFE 2016 International Conference on Physical Ergonomics and Human Factors, vol. 489 of Advances in Intelligent Systems and Computing, pp. 155-163, Springer, 2016.

[27] M. Kankaraš, G. Montt, M. Paccagnella, G. Quintini, and W. Thorn, Skills matter: further results from the survey of adult skills. oecd skills studies, OECD Publishing, 2016.

[28] M. A. Hentschel, M. L. Haaksma, and T. H. van de Belt, "Wearable technology for the elderly: Underutilized solutions," European Geriatric Medicine, vol. 7, no. 5, pp. 399-401, 2016.

[29] A. Rosales, M. Fernández-Ardèvol, F. Comunello, S. Mulargia, and N. Ferran-Ferrer, "Older people and smartwatches, initial experiences," El Profesional de la Información, vol. 26, no. 3, p. 457, 2017.

[30] J. E. Lewis and M. B. Neider, "Designing wearable technology for an aging population," Ergonomics in Design, vol. 25, no. 3, Article ID 1064804616645488, pp. 4-10, 2017.

[31] J. A. Batsis, J. A. Naslund, L. E. Gill, R. K. Masutani, N. Agarwal, and S. J. Bartels, "Use of a wearable activity device in rural older obese adults: a pilot study," Gerontology and Geriatric Medicine, vol. 2, Article ID 2333721416678076, 2016.

[32] K. Mercer, L. Giangregorio, E. Schneider, P. Chilana, M. Li, and K. Grindrod, "Acceptance of commercially available wearable 
activity trackers among adults aged over 50 and with chronic illness: a mixed-methods evaluation," JMIR mHealth and uHealth, vol. 4 , no. 1, 2016.

[33] K. Kunze, N. Henze, and K. Kise, "Wearable computing for older adults - Initial insights into head-mounted display usage," in Proceedings of the 2014 ACM International Joint Conference on Pervasive and Ubiquitous Computing, UbiComp 2014, pp. 83-86, September 2014.

[34] A. Jang, D. MacLean, and J. Heer, "BodyDiagrams: Improving communication of pain symptoms through drawing," in Proceedings of the 32nd Annual ACM Conference on Human Factors in Computing Systems, CHI 2014, pp. 1153-1162, New York, NY, USA, May 2014.

[35] T. Serif, G. Ghinea, and A. O. Frank, "Visualizing pain data for wheelchair users: a ubiquitous approach," J. Mob. Multimed, vol. 1, no. 2, pp. 161-177, June 2005.

[36] E. A. A. Jaatun, D. F. Haugen, Y. Dahl, and A. Kofod-Petersen, "Designing a reliable pain drawing tool: Avoiding interaction flaws by better tailoring to patients' impairments," Personal and Ubiquitous Computing, vol. 19, no. 3, article no. A010, pp. 635648, 2015.

[37] J. Follett, Designing for Emerging Technologies: UX for Genomics, Robotics, and the Internet of Things, O'Reilly Media, Inc, 2014.

[38] I. Rodriguez, C. Fuentes, V. Herskovic, and M. Campos, "BePain: A wearable interface to self-report pain and emotions," in Proceedings of the 2016 ACM International Joint Conference on Pervasive and Ubiquitous Computing, UbiComp 2016, pp. 11201125, New York, NY, USA, September 2016.

[39] B. A. Rosser, K. E. Vowles, E. Keogh, C. Eccleston, and G. A. Mountain, "Technologically-assisted behaviour change: A systematic review of studies of novel technologies for the management of chronic illness," Journal of Telemedicine and Telecare, vol. 15, no. 7, pp. 327-338, 2009.

[40] K. Lyons and H. Profita, "The multiple dispositions of on-body and wearable devices," IEEE Pervasive Computing, vol. 13, no. 4, pp. 24-31, 2014.

[41] Y.-M. Fang and C.-C. Chang, "Users' psychological perception and perceived readability of wearable devices for elderly people," Behaviour \& Information Technology, vol. 35, no. 3, pp. 225232, 2016.

[42] X. Liu, K. Vega, P. Maes, and J. A. Paradiso, "Wearability Factors for Skin Interfaces," in Proceedings of the the 7th Augmented Human International Conference 2016, pp. 1-8, New York, NY, USA, Feburary 2016.

[43] C. Cornelius, R. Peterson, J. Skinner, R. Halter, and D. Kotz, "A wearable system that knows who wears it," in Proceedings of the 12th Annual International Conference on Mobile Systems, Applications, and Services, MobiSys 2014, pp. 55-67, New York, NY, USA, June 2014.

[44] I. Rodríguez, C. Fuentes, V. Herskovic, and M. Campos, "Monitoring chronic pain: comparing wearable and mobile interfaces," in Lecture Notes in Computer Science, vol. 10069, pp. 234-245, 2016.

[45] A. Ferrari, "Digital competence in practice: an analysis of frameworks," Tech. Rep. 9, Research Centre of the European Commission, 2012.

[46] J. Brooke, "Sus-a quick and dirty usability scale," Usability Evaluation in Industry, vol. 189, no. 189, pp. 4-7, 1996.

[47] T. Tullis and W. Albert, Measuring the User Experience: Collecting, Analyzing, and Presenting Usability Metrics, Morgan Kaufmann Publishers Inc, San Francisco, CA, USA, 2008.
[48] V. Braun and V. Clarke, "Using thematic analysis in psychology," Qualitative Research in Psychology, vol. 3, no. 2, pp. 77-101, 2006.

[49] B. A. Rosser and C. Eccleston, "Smartphone applications for pain management," Journal of Telemedicine and Telecare, vol. 17, no. 6, pp. 308-312, 2011.

[50] G.-N. Gimhae, "Six human factors to acceptability of wearable computers," International Journal of Multimedia and Ubiquitous Engineering, vol. 8, no. 3, 2013.

[51] J. Rantakari, V. Inget, A. Colley, and J. Häkkilä, "Charting design preferences on wellness wearables," in Proceedings of the 7th Augmented Human International Conference, AH 2016, New York, NY, USA, February 2016.

[52] C. Buenaflor and H.-C. Kim, "Six human factors to acceptability of wearable computers," International Journal of Multimedia and Ubiquitous Engineering, vol. 8, no. 3, pp. 103-114, 2013.

[53] J. Van Heek, A. K. Schaar, B. Trevisan, P. Bosowski, and M. Ziefle, "User requirements for wearable smart textiles. Does the usage context matter (medical vs. sports)?" in Proceedings of the 8th International Conference on Pervasive Computing Technologies for Healthcare of Pervasive Healthcare (PervasiveHealth '14), pp. 205-209, ICST, Brussels, Belgium, 2014.

[54] A. Pyae, T. Liukkonen, T. Saarenpää, M. Luimula, P. Granholm, and P. Smed, "When japanese elderly people play a finnish physical exercise game: a usability study," The Journal of Usability Studies, vol. 11, no. 4, pp. 131-152, 2016.

[55] F. H. A. Razak, R. Sulo, and W. A. W. Adnan, "Elderly user mental model of reminder system," in Proceedings of the 10th Asia-Pacific Conference on Computer-Human Interaction, APCHI 2012, pp. 193-200, New York, NY, USA, August 2012.

[56] J. Isleifsdottir and M. Larusdottir, "Measuring the user experience of a task oriented software," in Proceedings of the International Workshop on Meaningful Measures: Valid Useful User Experience Measurement, pp. 97-101, 2008.

[57] ISO, "Ergonomics of human system interaction - part 210: human-centred design for interactive systems," Tech. Rep. SO DIS 9241-210, International Organization for Standardization, Genève, Switzerland, 2010.

[58] J. McCarthy and P. Wright, “Technology as experience," Interactions, vol. 11, no. 5, pp. 42-43, 2004.

[59] R. D. Ellis and J. C. Allaire, "Modeling computer interest in older adults: The role of age, education, computer knowledge, and computer anxiety," Human Factors, vol. 41, no. 3, pp. 345-355, 1999.

[60] S. Kumar, L. C. II. Ureel, H. King, and C. Wallace, "Lessons from our elders: Identifying obstacles to digital literacy through direct engagement," in Proceedings of the 6th ACM International Conference on PErvasive Technologies Related to Assistive Environments, PETRA 2013, New York, NY, USA, May 2013.

[61] J. M. Nassar, K. Mishra, K. Lau, A. A. Aguirre-Pablo, and M. M. Hussain, "Recyclable Nonfunctionalized Paper-Based Ultralow-Cost Wearable Health Monitoring System," Advanced Materials Technologies, vol. 2, no. 4, Article ID 1600228, 2017. 

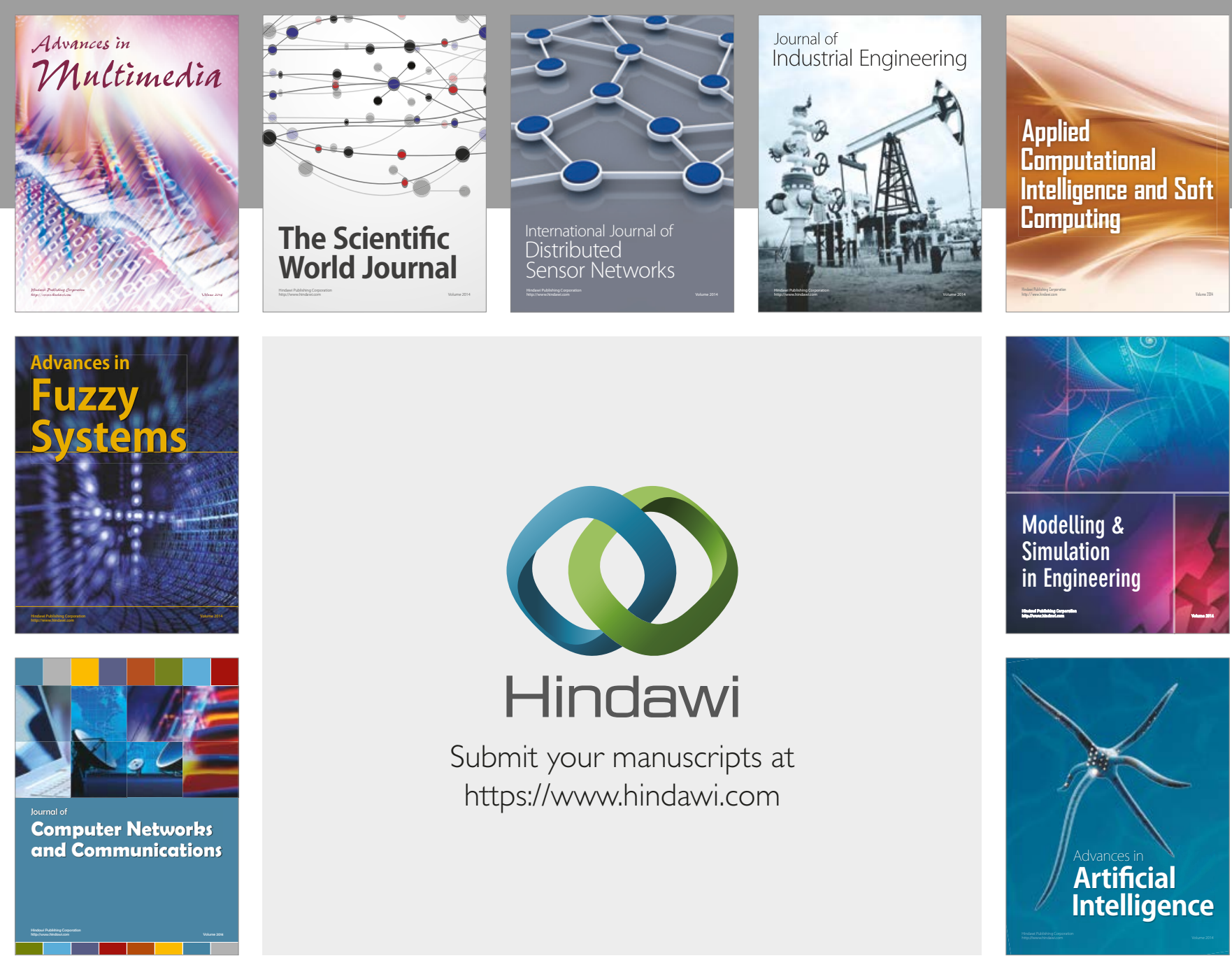

\section{Hindawi}

Submit your manuscripts at

https://www.hindawi.com
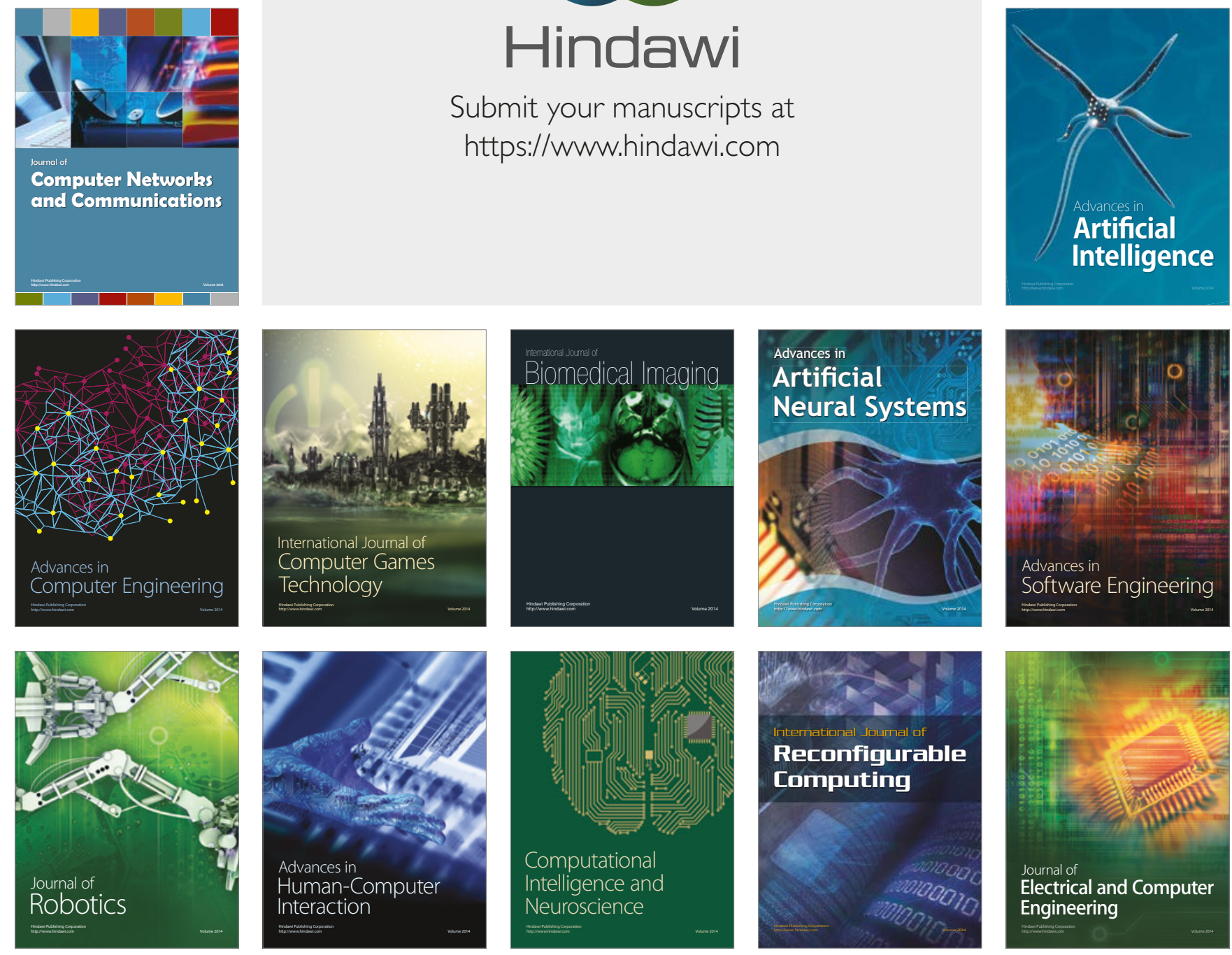